\title{
Monte Carlo simulations of microchannel plate detectors I: steady-state voltage bias results
}

\author{
Ming Wu, ${ }^{\text {a) }}$ Craig A. Kruschwitz, Dane V. Morgan, and Jiaming Morgan \\ National Security Technologies, 182 East Gate Drive, Los Alamos, New Mexico \\ 87544
}

X-ray detectors based on straight-channel microchannel plates (MCPs) are a powerful diagnostic tool for two-dimensional, time-resolved imaging and timeresolved x-ray spectroscopy in the fields of laser-driven inertial confinement fusion and fast z-pinch experiments. Understanding the behavior of microchannel plates as used in such detectors is critical to understanding the data obtained. The subject of this paper is a Monte Carlo computer code we have developed to simulate the electron cascade in a microchannel plate under a static applied voltage. Also included in the simulation is elastic reflection of low-energy electrons from the channel wall, which is important at lower voltages. When model results were compared to measured microchannel plate sensitivities, good agreement was found. Spatial resolution simulations of MCP-based detectors were also presented and found to agree with experimental measurements. [PACS code: $07.05 . \mathrm{Tp}]$

a) Electronic mail: WuM@,nv.doe.gov 


\section{INTRODUCTION}

Microchannel plate detectors are widely used as electron, ion, and x-ray detectors, as well as imaging tools in many areas of scientific research. Their principles of operation have been documented in the literature,,$^{1,2,3,4}$ as have extensive research efforts to characterize detection sensitivity, ${ }^{5,6,7}$ angular and energy dependence, ${ }^{8,9,10,11}$ and temporal and spatial resolution. ${ }^{12,13}$ In many previous studies, a discrete dynode gain model has been used to describe the MCP gain dependence on the applied voltage. ${ }^{14}$ This dependence is extremely nonlinear. The discrete dynode model assumes that the MCP can be treated as a conventional, discrete-stage electron multiplier with a fixed number of stages. This gain model uses a few free parameters, which are chosen to best fit the data for a particular MCP. The discrete dynode model seems to work well to describe the MCPs' behavior under some circumstances, but several factors are not included when inferring the secondary electron yield from gain measurements. For example, the discrete dynode model assumes that the number of dynode stages is independent of the applied voltage on the MCP (indeed, the number of stages, $n$, is chosen to best fit the gain vs. voltage data), which is unlikely to be valid.

In addition to the discrete dynode model, previous researchers have also performed Monte Carlo-based computer simulations of the MCP response to a steady-state voltage for straight and titled microchannels. ${ }^{15,16,17,18}$ These simulations apparently did not include the constraint of energy conservation for the secondary electrons. This constraint prevents the aggregate energy of the emitted electrons from exceeding the primary electron energy. Furthermore, these previous efforts omit the effects of low-energy 
electrons' elastic scattering from the channel walls, potentially an important effect for the low-impact energies prevalent in an $\mathrm{MCP}$ electron cascade. A further difficulty encountered by all such previous efforts (and, indeed, the present one) is that a fair number of adjustable parameters in these simulations cannot be unambiguously determined from the existing data. Consequently, inconsistent parameter sets appear in the literature, with some more robustly constrained by the data than others.

In the last two decades, high-speed, gated MCP x-ray detectors have proven to be a powerful diagnostic tool for two-dimensional, time-resolved imaging and time-resolved x-ray spectroscopy in the field of laser-driven inertial confinement fusion and fast z-pinch experiments. ${ }^{19,20,21}$ These detectors quantitative measurements are critical for a comprehensive understanding of the experimental results. To assist their characterizations and to aid design improvements, a more comprehensive Monte Carlo simulation model for the MCP detector is needed.

The main purpose of this paper is twofold. First, we describe our new simulation model, which includes the effects of elastically scattered electrons and precludes the total secondary electron energy from exceeding that of the primary. Then, we compare the simulations with experimental results to obtain a reasonable set of model parameters for the MCP. Although these parameters can't be uniquely determined, achieving good agreement between the simulation and experiments in DC operations can lead to a useful parameter set to study MCP performance with subnanosecond high-voltage pulses.

In section II, we present a Monte Carlo simulation model and computational algorithm. Comparisons of simulated and measured MCP gain appear in section III. 
Spatial resolution of the MCP detector is discussed in section IV. Section V summarizes our results.

\section{MODEL AND COMPUTATIONAL ALGORITHM}

A microchannel plate is essentially an array of parallel continuous electron multipliers. Individual MCP channels are typically $\sim 10 \mu \mathrm{m}$ in diameter, and the plates themselves are usually $450-600 \mu \mathrm{m}$ thick. Generally, the channels are set at an angle, $\alpha$, relative to the MCP surface normal, also called the "bias angle." The MCP is also characterized by the ratio of its thickness to the channel diameter, or its L/D ratio.

MCP electron cascade dynamics can be approximated by the behavior of a single microchannel. For our purposes, it is acceptable to assume that all microchannels in a particular plate are identical because we are neglecting any cross-talk effects between adjacent channels. When the MCP is operated at high count rates or hard x-ray detection, the effects of neighbor channels can be more significant. ${ }^{22,23}$ The cascade is taken to be initiated by some number of incident electrons. Their number, incident energy, initial direction, and initial location are determined by the user. For the simulations presented in this paper, initial positions are all at the MCP input face. For each electron incident on the channel wall, equations dependent on the incident energy and angle are used to determine the mean secondary emission yield. The incident energy, $V_{m}\left(\theta_{i}\right)$, at which an electron colliding with the channel wall will produce the maximum yield is given as

$$
V_{m}\left(\theta_{i}\right)=\frac{V_{m}(0)}{\sqrt{\cos \theta_{i}}}
$$


where $V_{m}(0)$ is the incident energy producing maximum yield at normal incidence, and $\theta_{i}$ is the incident angle. ${ }^{24}$ Values for $V_{m}(0)$ found in the literature for lead glass run from $\sim 200$ to $300 \mathrm{eV} .{ }^{25} \mathrm{We}$ discovered that using a value near the middle of this range (260$270 \mathrm{eV}$ ) achieved a good fit with our experimental data.

The maximum yield for a primary electron incident at angle $\theta_{i}$ is

$$
\delta_{m}\left(\theta_{i}\right)=\delta_{m}(0) \exp \left[\alpha\left(1-\cos \theta_{i}\right)\right],
$$

where $\alpha$ is a material-dependent parameter determining the variation of the maximum yield with incident angle, and $\delta_{m}(0)$ is the maximum yield at normal incidence. ${ }^{24}$ Typical values for $\alpha$, for lead glass used in constructing MCPs, range from 0.4 to $0.6 .{ }^{25} \mathrm{We}$ begin our simulation with a value of 0.5 , then adjust to fit the experimental data. Values for $\delta_{m}(0)$ for MCP lead glass typically range between $\sim 3.0$ and 4.0. In the simulations presented here, $\delta_{m}(0)$ is taken to be 4.0 .

The actual value of the average secondary emission for a primary electron incident on the MCP channel surface at angle $\theta_{i}$ and with energy $V_{i}$ is determined by ${ }^{26}$

$$
\delta\left(V_{i}, \theta_{i}\right)=\delta_{m}\left(\theta_{i}\right) \frac{s \frac{V_{i}}{V_{m}\left(\theta_{i}\right)}}{s-1-\left(\frac{V_{i}}{V_{m}\left(\theta_{i}\right)}\right)^{s}},
$$

where $s$ is an adjustable, material-dependent parameter $>1$, typically around 1.3 (the value used in this work). The actual secondary yield is determined by random sampling of a Poisson distribution with a mean value of $\delta\left(V_{i}, \theta_{\mathrm{i}}\right)$. Other authors have used a variety 
of alternate forms for Eq. (3), each with their own adjustable parameters. We have found there to be little difference between the performances of these models for simulating the secondary emission processes in a microchannel plate. In large measure, this is due to the fact that the majority of electron-channel wall collisions are low energy $(<100 \mathrm{eV})$, in a domain in which each of these models is nearly linear.

With the number of secondary electrons determined, each electron's initial energy is assigned by sampling a Maxwell-Boltzmann probability distribution

$$
P\left(E_{s}\right)=C \frac{E_{s}}{E_{0}} \exp \left(-\frac{E_{s}}{E_{0}}\right),
$$

where $E_{0}$ is the most probable secondary energy, and $C$ is a normalization constant. Experimental data for MCP-type lead glass indicate a value of about $2-3 \mathrm{eV}$ for $E_{0 .}{ }^{25,27,28}$ This work uses a value of $3 \mathrm{eV}$. It should be noted that various forms for the secondary energy distribution have been used by different authors. We have tried several, and found that the specific form of the distribution generally has little effect on the simulation results. Of greatest importance to the outcome is the value of the most probable energy. While we assume the emission energies of the secondary electrons to be uncorrelated, we do require that energy is conserved by ensuring that the sum of the secondary electron emission energies be less than the impact energy of the primary electron. We implement this by repeatedly sampling the secondary electron energies from the above MaxwellBoltzmann distribution until the conservation condition is met.

The direction in which each secondary electron is emitted is sampled from a cosine distribution,

$$
P(\theta) \propto \cos (\theta)
$$


where $\theta$ is the angle relative to the surface normal. We assume that the emission angles of the secondary electrons are fully uncorrelated, independent of the incident energy and angle, and uncorrelated with the emission energies. Experiments have shown these to be reasonable assumptions. ${ }^{24}$

The trajectories of the secondary electrons are then calculated using nonrelativistic equations of motion. Since the maximum electron energies are on the order of $1 \mathrm{keV}$, a small fraction of the rest-mass energy, the problem can safely be treated nonrelativistically. After the electron equations of motion are solved, the impact energy and angle of the electron with the channel wall are determined, giving the initial conditions for the next generation of electrons. This process is repeated until the electrons emerge from the output end of the channel or the cascade dies out, yielding no output electrons.

In this simple model, we assume that only the photoelectrons and Auger electrons generated adjacent to the microchannel can initiate a cascade within the channel, which agrees with experimental observation. ${ }^{10}$ From the E-field calculations ${ }^{29}$, the photoelectrons produced in the field region would not be further amplified and would no contribute to the total signal. The initial photoelectrons are generated in a timeframe $\left(<10^{-12} \mathrm{~s}\right)$ that is much shorter than the transit time of the cascading electrons. The time lag of secondary emission is estimated to be $10^{-13}$ to $10^{-14} \mathrm{~s}$, which is much shorter than any timescale relevant to electron-cloud effects. ${ }^{30}$ Thus, we assume that the secondary electrons are generated instantaneously when a primary electron hits the lead glass surface. 
In previous Monte Carlo simulations, ${ }^{15,16,17,18}$ the possibility of the incoming electrons' elastic reflection was neglected. In our simulations, we found elastic scattering to be an important effect, particularly for low-bias voltages. It was necessary in order to use one set of parameters to describe the gain variation of the MCP with bias voltage over the full range of voltages of interest. The probability of electron reflections from lead glass at normal incidence as a function of energy was studied by Scholtz et al. ${ }^{27}$ They discovered that for $10-\mathrm{eV}$ primary electrons incident normal to a lead glass surface, $\sim 95 \%$ of the resulting secondary electrons were in fact elastically reflected primary electrons, decreasing to $\sim 5 \%$ for $100-\mathrm{eV}$ primary electrons. Scholtz et al. ${ }^{18}$ found that the following equation produced a good fit to the reflected fraction of secondary electrons vs. primary electron energy data:

$$
f_{0}=\exp \left(1.59+3.75 \ln \left(E_{p}\right)-1.37\left[\ln \left(E_{p}\right)\right]^{2}+0.12\left[\ln \left(E_{p}\right)\right]^{\beta}\right) / 100 .
$$

Unfortunately, the research did not examine any angular dependence for this effect. Nevertheless, we have included the option of using Scholtz's results in the simulations as an elastic scattering approximation, assuming the effect to be independent of incident angle and providing for an adjustable scaling factor, $R$, to be included in order to better fit our experimental data. Thus, the reflected fraction of secondaries is: $f=R \times f_{0}$.

Using Eq. (6) to find the reflected fraction of secondary electrons and Eq. (3) to find the true secondary yield, the average total yield (reflected primary electrons plus secondary electrons) is given by 


$$
\delta_{t}=\frac{\delta_{s}}{1-f+\delta_{s} f},
$$

where $\delta_{s}$ is the true secondary yield, and $f$ is given by Eq. (6). It then follows that the average reflected yield is

$$
\delta_{e}=f \delta_{t}
$$

Eq. (8) is used to determine the probability that an electron is elastically reflected after a collision with the channel wall. If reflection occurs, then the axial and angular components of the electrons' velocity are left unchanged, while the radial component is reversed. If reflection does not occur, the secondary electron yield and initial properties of the secondary electrons are determined, as described above.

\section{DC VOLTAGE SIMULATIONS}

Although these simulations can be adapted to MCPs with almost any geometry, we have concentrated most of our modeling efforts on an MCP with a 10- $\mu \mathrm{m}$ channel diameter and a thickness of $0.46 \mathrm{~mm}(\mathrm{~L} / \mathrm{D}=46)$. The parameters used in these simulations are summarized in Table 1. For MCPs with these characteristics, we have a large amount of experimental data that can be compared to our simulation results. Figure 1 shows simulation results for an MCP with the parameters shown in Table 1, with a steady-state bias of $-1000 \mathrm{~V}$. The simulation was initiated by introducing three electrons near the input end of the MCP, typical of what might be expected from a $1-\mathrm{keV}$ x-ray. The gain histogram for 2,000 separate runs shows that the average gain is about $1.2 \times 10^{4}$, but a considerable spread in the gain clearly exists. This is a consequence of the statistical nature of the secondary emission process. There is a clear indication of a peak in the gain histogram near 5000. The transit time distribution for these runs looks essentially 
Gaussian, with a mean transit time of 182 ps and a full-width half-maximum (FWHM) transit-time spread (TTS) of 54 ps. We lack transit-time measurements for the MCPs we are simulating (such measurements are difficult to make), but 200-ps transit times are consistent with existing measurements. ${ }^{16}$

Figure 2 shows simulation results for an identical MCP, but with an applied voltage bias of $-600 \mathrm{~V}$ DC. The average gain is 77 , more than two orders of magnitude lower than for the $-1000 \mathrm{~V}$ DC simulations, and the gain histogram lacks any indication of a peak. The lower gain is the result of smaller electron impact energies, which in turn result in smaller secondary electron yields. The mean transit time is $205 \mathrm{ps,} 20$ ps longer than for the $-1000 \mathrm{~V}$ DC simulations. This is a consequence of the decreased acceleration in the $-600 \mathrm{~V}$ DC bias case and the increased number of electron cascade generations. The electrons travel a shorter distance down the channel between collisions, and thus require more time to reach the output end. Also, the TTS for the $-600 \mathrm{~V}$ case is $74 \mathrm{ps}$, significantly longer than that of the $-1000 \mathrm{~V}$ DC case. This is because the spread in secondary electron energy and direction play a greater role at lower bias voltages, where the electrons travel shorter distances between collisions and impact the channel wall with lower energy.

In order to check the validity of our simulation results, we compared the modeled versus measured $\mathrm{MCP}$ sensitivities. The detector consists of an MCP with a thin conductive layer on the input (bias) and output (ground) surfaces, a phosphor screen coated on a fiber-optic faceplate at positive potential, and a coherent fiber bundle coupled between the faceplate and a charge-coupled device (CCD). Purchased from Burle, Inc., the MCPs had an L/D ratio of $46(\mathrm{~L}=460 \mu \mathrm{m}, \mathrm{D}=10 \mu \mathrm{m})$, with a channel bias angle of 
$8^{\circ}$. Both MCP surfaces were coated using physical vapor deposition at angles of $60^{\circ}$ and $45^{\circ}$ from the surface normal, respectively. They were coated in four separate layers with the following materials and thicknesses: $75 \AA \mathrm{Cr}, 5000 \AA \mathrm{Cu}, 75 \AA \mathrm{Cr}, 1000 \AA \mathrm{Au}$. The back surface was coated uniformly. The front surface was coated in six separate strips, each $4 \mathrm{~mm}$ wide $\times 40 \mathrm{~mm}$ long, separated by $2 \mathrm{~mm}$.

A negative bias voltage was applied to each strip, and the phosphor-coated fiber-optic faceplate was held at $+3000 \mathrm{~V}$ with respect to the MCP back surface. The coherent fiber bundle is $36 \mathrm{~mm}$ high $\times 40 \mathrm{~mm}$ wide and $48^{\prime \prime}$ long. The glass fiber is $10 \mu \mathrm{m}$ in diameter, and the overall quality area is $34 \times 38 \mathrm{~mm}$. The CCD camera is a Spectral Instruments 800 series with a KAF-16801E class 2 chip. The KAF-16801E is a high-performance, monochrome area, CCD image sensor with $4096 \mathrm{H} \times 4096 \mathrm{~V}(9-\mu \mathrm{m})$ photo-active pixels.

Gain and resolution experiments were performed on a Manson x-ray source with two symmetrical lines of sight. An Amptek XR-100-CZT x-ray pulse-height spectrometer was placed on the reference line of sight to record Manson source output, while the MCP was placed on the main line of sight at $\sim 2.7 \mathrm{~m}$ from the Manson source to obtain a uniform x-ray flux on all of the strips. Two Uniblitz x-ray shutters were installed on each line of sight to ensure equal x-ray exposures. The Manson source was operated at $8 \mathrm{kV}$ with $0.3 \mathrm{~mA}$ of emission current using silver and aluminum anodes, which have emission peaks at about 1.5 and $3.0 \mathrm{keV}$, respectively. Beryllium filters were used on both lines of sight to block light emission from the filament. The x-ray flux was controlled to be less than 1 quantum/microchannel/sec to prevent MCP saturation. Relative sensitivities were measured as a function of voltage for potentials ranging from 450 to $950 \mathrm{~V}$, increased in $50-\mathrm{V}$ increments. 
Both modeled and measured sensitivities are plotted versus voltage in Figure 3. The simulated gains have been scaled so that the model value at $450 \mathrm{~V}$ is set to the average of the measured data. Clearly the model reproduces the trend in the measured data extremely well over virtually the entire voltage range. However, the data show some leveling off at $950 \mathrm{~V}$, which is not seen in the simulations. This leveling off is due to the onset of saturation in the CCD used to collect the data, and is not an effect of the MCP.

\section{SPATIAL RESOLUTION}

The three-dimensional electron transport model also allows us to predict MCP resolution. Resolution calculations were based on the set of parameters for the MCP camera back imaging system described in the experiments. The electron position distribution is obtained by calculating the ballistic trajectory of the MCP output electrons hitting the phosphor plate held at a fixed positive potential. The phosphor is located 0.029 in. from the MCP exit face, which is at ground potential. Experimental measurements of the detector's spatial resolution were made using a knife-edge resolution target. For some simulations, we assumed that the conductive gold coating extended into the channel output a distance of about 1.5 channel diameters, or $15 \mu \mathrm{m}$. This "end-spoiling" created an electron focusing effect, which has been detailed previously. ${ }^{14,31,32,33}$

For our resolution calculations, we have generated a set of output electrons from a 729-channel array in a hexagonal-packed geometry with a center-to-center distance of $12 \mu \mathrm{m}$. An orthogonal x-y coordinate system was established in the phosphor plane with the bias angle along the y axis. The resulting model-generated image was rectangular, 
with the $\mathrm{x}$ dimension shorter than the $\mathrm{y}$ dimension by a factor of 0.866 . The resolution was calculated for bias angles of 5.0, 8.0, and 12.0 degrees, to determine how this parameter affected resolution. We also calculated the effects of changing the phosphor plate potential with respect to the MCP exit face for each bias angle. The simulated distributions along $\mathrm{x}$ and $\mathrm{y}$ axes are shown in Figure 4(a). Here we can see the $\mathrm{x}$ axis distribution is symmetrical and centered. However, electron distribution along the y axis is asymmetrical and off-center, as expected. This asymmetry is reduced when the bias voltage is increased.

Figure 4(b) shows the linespread function (LSF), which is the first derivative of the electron distribution function shown in Figure 4(a). The FWHM of each LSFs was calculated for phosphor potentials $V_{p h}$ ranging from $500 \mathrm{~V}$ to $4000 \mathrm{~V}$. The resolution in the $\mathrm{x}$ and $\mathrm{y}$ directions are plotted in Figure 5 as a function of $V_{p h}^{-1 / 2}$. A simple analytical expression for the spatial resolution $\delta$ of an MCP detector ${ }^{4}$ indicates that the relationship between $\delta$ and $V_{p h}$ is $\delta \propto V_{p h}^{-1 / 2}$, and therefore, such a plot should yield a straight line.

From Figure 5, it is apparent that such a relationship does in fact hold, in both the $\mathrm{x}$ and y directions. Figure 6 shows the LSF FWHM as a function of $V_{p h}$ along the $\mathrm{x}$ and $\mathrm{y}$ axes for different MCP bias angles. Clearly, bias angle has no effect on the spatial resolution in the $\mathrm{x}$ direction. Along the $\mathrm{y}$ axis, however, we observe reduced resolution as a result of the MCP bias angle, with increasing bias angles leading to reduced resolution.

Figure 7 compares the experimental measurements of the spatial resolution of the MCP with CCD detector system and the simulations of the MCP spatial resolution. Experimental measurements and simulations have been performed for $V_{p h}$ between 500 and $4000 \mathrm{~V}$. The resolution target was place in front of MCP with a spacing less than 1 
$\mathrm{mm}$. In our measurements, the detector was placed 2.7 meter away from the Manson source. The $1 \mathrm{~mm}$ spacing between the resolution target and the MCP should have almost no effect on measured line spread function. For the simulated resolutions, 37- $\mu \mathrm{m}$ instrument resolution broadening was added in quadrature to the simulated LSF FWHM, and the $\mathrm{x}$ and $\mathrm{y}$ FWHM were averaged. The measured and simulated results showed excellent agreement. Additionally, Figure 7 shows a single point at a 2-kV phosphor bias, the result of including the effects of end-spoiling in the simulation. The outcome is a reduction in the LSF FWHM, and hence, an improvement in spatial resolution.

\section{CONCLUSIONS}

We have discussed our new Monte Carlo simulation model, developed as a tool for assisting with the design of high speed, gated x-ray cameras and for interpreting the data obtained from such devices. The code uses a fairly standard set of equations for determining secondary emission yields, based in part on experimental data obtained with MCP lead glass. It also takes into account elastic reflections of low-energy electrons from the channel wall and requires that the total secondary electron energy not exceed the energy of the parent primary electron.

The large amount of experimental data we have for $0.46-\mathrm{mm}$-thick, 10 - $\mu \mathrm{m}$ porediameter MCPs allows us to test our simulation code and fine-tune unknown MCP secondary emission parameters. With this fine-tuning, our simulations of gain vs. DC bias voltage and of MCP spatial resolution achieve excellent agreement with our experimental data. 
Future work will involve extending the MCP simulations to include subnanosecond pulsed voltages. We also plan to study MCP saturation due to space charge, positive wall charge buildup, and strip current limitations. These topics will be the subjects of future publications.

\section{ACKNOWLEDGMENTS}

This research has been partially supported by the NSTec Nevada Test Site-Directed Research, Development, and Demonstration (SDRD) and SNL Above-Ground Experimentation (AGEX) programs.

This manuscript has been authored by National Security Technologies, LLC, under Contract No. DE-AC52-06NA25946 with the U.S. Department of Energy. The United States Government retains and the publisher, by accepting the article for publication, acknowledges that the United States Government retains a non-exclusive, paid-up, irrevocable, world-wide license to publish or reproduce the published form of this manuscript, or allow others to do so, for United States Government purposes. 
${ }^{1}$ J. L. Wiza, Nucl. Instrum. Methods 162, 587 (1979).

${ }^{2}$ G. W. Fraser, M. A. Barstow, M. J. Whiteley, and A. Wells, Nature (London) 300, 509 (1982).

${ }^{3}$ G. W. Fraser, M. A. Barstow, J. F. Pearson, M. J. Whiteley, and M. Lewis, Nucl. Instrum. Methods 224, 272 (1984).

${ }^{4}$ J. D. Kilkenny, Laser Part. Beams 9, 49 (1990).

${ }^{5}$ Frederic Ze, Otto L. Landen, Perry M. Bell, Robert E. Turner, Teresa Tutt, Sharon S. Alvarez, and Robert L. Costa, Rev. Sci. Instrum. 70, 659 (1999).

${ }^{6}$ O. L. Landen, P. M. Bell, J. A. Oertel, J. J. Satariano, and D. K. Bradley, SPIE 2002, 2 (1993).

${ }^{7}$ O. L. Landen, A. Abare, B. A. Hammel, P. M. Bell, and D. K. Bradley, SPIE 2273, 245 (1993).

${ }^{8}$ M. Hirata, T. Cho, E. Takahashi, N. Yamaguchi, T. Kondoh, K. Matsuda, S. Aoki, K. Tanaka, H. Maezawa, and S. Miyoshi, Nucl. Instrum. Methods Phys. Res. B 66, 479 (1992).

${ }^{9}$ O. L. Landen, A. Lobban, T. Tutt, P. M. Bell, R. Costa, D. R. Hargrove, and F. Ze, Rev. Sci. Instrum. 72, 709 (2001).

${ }^{10}$ G. A. Rochau, J. E. Bailey, G. A. Chandler, T. J. Nash, D. S. Nielsen, G. S. Dunham, O. F. Garcia, N. R. Joseph, J. W. Keister, M. J. Madlener, D. V. Morgan, K. J. Moy, and M. Wu, Rev. Sci. Instrum. 77, 10E323 (2006).

${ }^{11}$ N. Yamaguchi, T. Cho, T. Kondoh, M. Hirata, and S. Miyoshi, Rev. Sci. Instrum 60, 368 (1989).

${ }^{12}$ H. F. Robey, K. S. Bundil, and B. A. Remington, Rev. Sci. Instrum. 68, 792 (1997).

13 C. J. Pawley and A. V. Deniz, Rev. Sci. Instrum. 71, 1286 (2000).

${ }^{14}$ P. Eberhardt, Appl. Opt. 18, 1418 (1979).

${ }^{15}$ A. J. Guest, Acta Electronica 14, 79 (1971).

${ }^{16}$ M. Ito, H. Kume, and K. Oba, IEEE Trans. Nucl. Sci. NS-31, 408 (1984). 
${ }^{17}$ Y. S. Choi and J. M. Kim, IEEE Trans. Elec. Dev. 47, 1293 (2000).

${ }^{18}$ G. J. Price and G. W. Fraser, Nucl. Instrum. Methods Phys. Res. A 474, 188 (2001).

${ }^{19}$ T. McCarville, S. Fulkerson, R. Booth, J. Emig, B. Young, S. Anderson, and B. Heeter, Rev. Sci. Instrum. 76, 103501 (2005).

${ }^{20}$ J. A. Oertel, R. Aragonez, T. Archuleta, C. Barnes, L. Casper, V. Fatherley, T. Heinrichs, R. King, D. Landers, F. Lopez, P. Sanchez, G. Sandoval, L. Schrank, P. Walsh, P. Bell, M. Brown, R. Costa, J. Holder, S. Montelongo, and N. Pederson, Rev. Sci. Instrum. 77, 10E308 (2006).

${ }^{21}$ J. E. Bailey, G. A. Chandler, S. A. Slutz, I. Golovkin, P. W. Lake, J. J. MacFarlance, R. C. Mancini, T. J. Burris-Mog, G. Cooper, R. J. Leeper, T. A. Mehlhorn, T. C. Moore, T. J. Nash, D. S. Nielsen, and C. L. Ruiz, Phys. Rev. Lett. 92, 85002 (2004).

${ }^{22}$ G. W. Fraser, M. T. Pain, and J. E. Lees, Nucl. Instrum. Meth. in Phys. Research A327, 328 (1993).

${ }^{23}$ P. M. Shikhaliev, Nucl. Instrum. Meth. in Phys. Research A398, 229 (1997).

${ }^{24}$ H. Bruining, Physics and Applications of Secondary Electron Emission (Pergamon Press, London, 1954).

${ }^{25}$ A. Authinarayanan and R. W. Dudding, Adv. Electron. Electron Phys. A 40, 167 (1976).

${ }^{26}$ M. A. Furman and M. T. F. Pivi, Phys. Rev. ST AB 5, 124404-1 (2002).

${ }^{27}$ J. J. Scholtz, D. Dijkkamp, and R. W. A. Schmitz, Philips. J. Res. 50, 375 (1996).

${ }^{28}$ G. E. Hill, Adv. Electron. Electron Phys. A 40, 153 (1976).

29 E. Gatti, K. Oba, and P. Rehak, IEEE Trans. Nucl. Sci. NS-30, 461 (1983).

${ }^{30}$ A. J. Dekker, in Solid State Physics, edited by F. Seitz (Academic Press, New York, 1958), Vol. 6, Chap. Secondary Electron Emission, p. 215.

${ }^{31}$ I. M. Bronshteyn, A. V. Yevdokimov, V. M. Stozharov, and A. M. Tyutikov, Radio Eng. Electron. Phys. 24, 150 (1979).

${ }^{32}$ Nobuyoshi Koshida and Masato Hosobuchi, Rev. Sci. Instrum. 56, 1329 (1985). 
${ }^{33}$ Nobuyoshi Koshida, Rev. Sci. Instrum. 57, 354 (1986). 
Table 1. MCP parameters used in Monte Carlo simulations

\begin{tabular}{|c|c|}
\hline MCP Parameter & Value \\
\hline L/D & 46 \\
\hline $\mathrm{D}$ & $10 \mu \mathrm{m}$ \\
\hline$\Theta($ bias angle $)$ & $8^{\circ}$ \\
\hline$V_{m}(0)$ & $260 \mathrm{eV}$ \\
\hline$\delta_{m}(0)$ & 4.0 \\
\hline$\alpha$ & 0.6 \\
\hline$E_{0}$ & $3 \mathrm{eV}$ \\
\hline$R$ & 0.85 \\
\hline
\end{tabular}


Figure 1. Simulation results for an $\mathrm{L} / \mathrm{D}=46,10-\mu \mathrm{m}$ pore-diameter $\mathrm{MCP}$, biased at $1000 \mathrm{~V} \mathrm{DC}$, with a bias angle of $\alpha=8^{\circ}$ : (a) gain histograms. (b) Transit-time distribution.

Figure 2. Simulation results for an $\mathrm{L} / \mathrm{D}=46,10-\mu \mathrm{m}$ pore-diameter $\mathrm{MCP}$, biased at $600 \mathrm{~V}$ DC, with a bias angle of $\alpha=8^{\circ}$ : (a) gain histograms. (b) Transit-time distribution.

Figure 3. Comparison of simulated and measured MCP relative sensitivity vs. DC voltage.

Figure 4 (a). Electron distribution on the phosphor along the $\mathrm{x}$ and $\mathrm{y}$ axes, with an $8^{\circ}$ bias angle and 3000-V phosphor potential. (b) Linespread function along the $\mathrm{x}$ and $\mathrm{y}$ axes. The peaks along the $y$ axis broaden when the bias angle is increased.

Figure 5. Resolution as a function of phosphor potential along the $\mathrm{x}$ axis, which is perpendicular to the bias angle, and along the y axis, parallel to the bias angle.

Figure 6. FWHM linespread function for different MCP bias angles: (a) linespread function along axis perpendicular to bias angle. (b) LSF along axis parallel to bias angle.

Figure 7. Comparison of simulated and measured MCP camera system spatial resolution. 

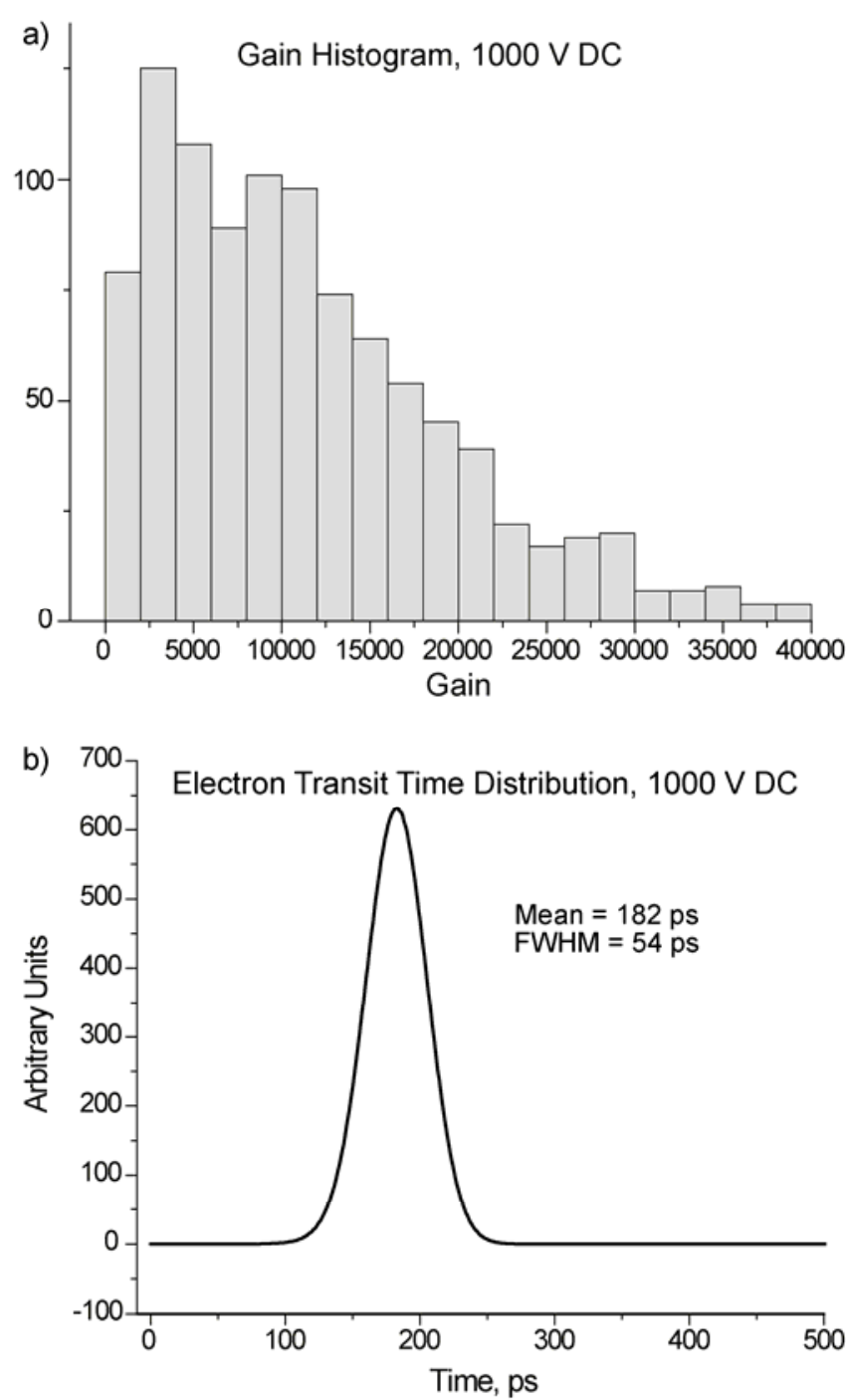

Figure 1. Wu 

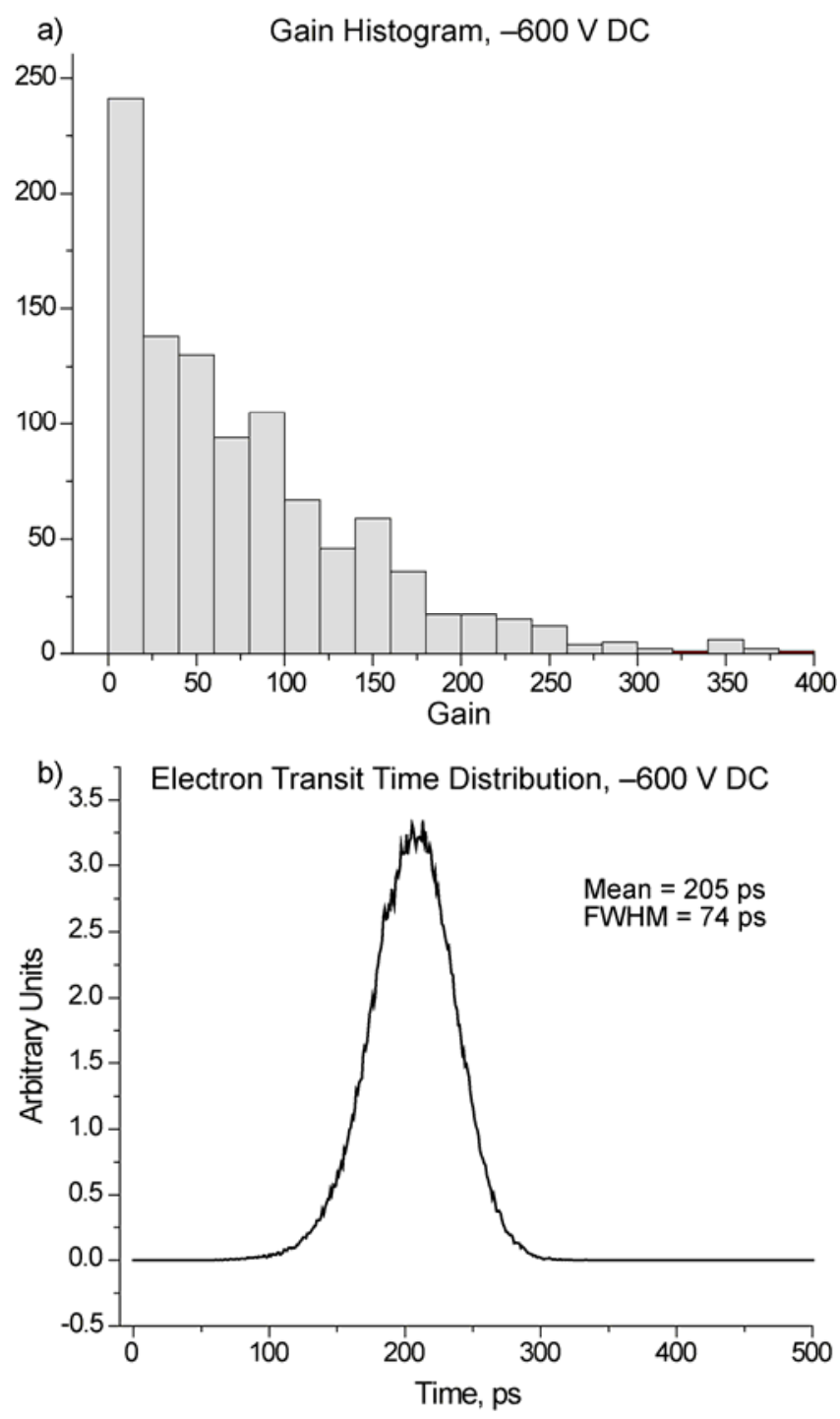

Figure 2. Wu 


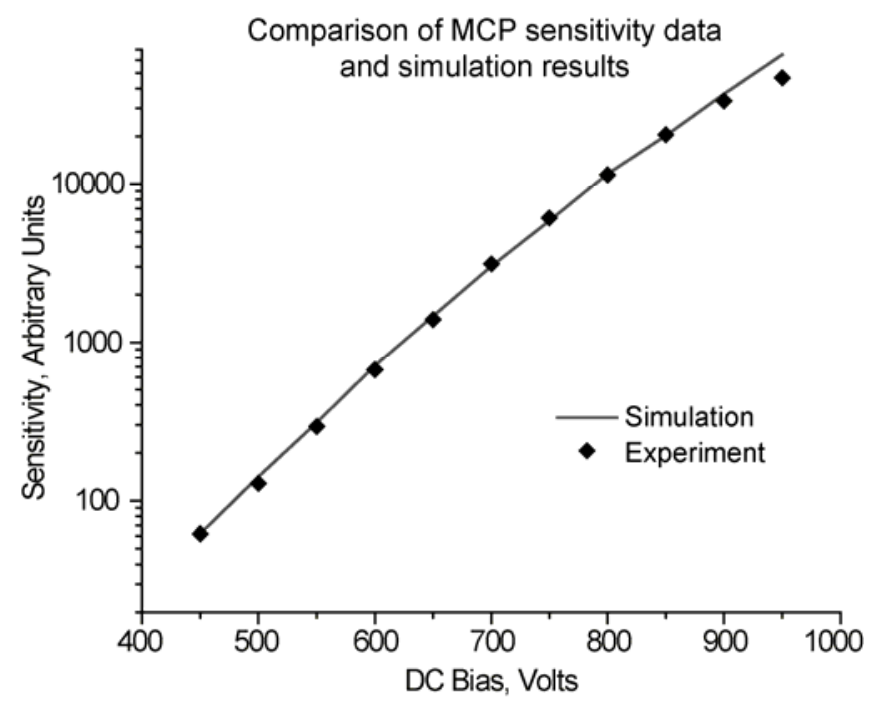

Figure 3. Wu 

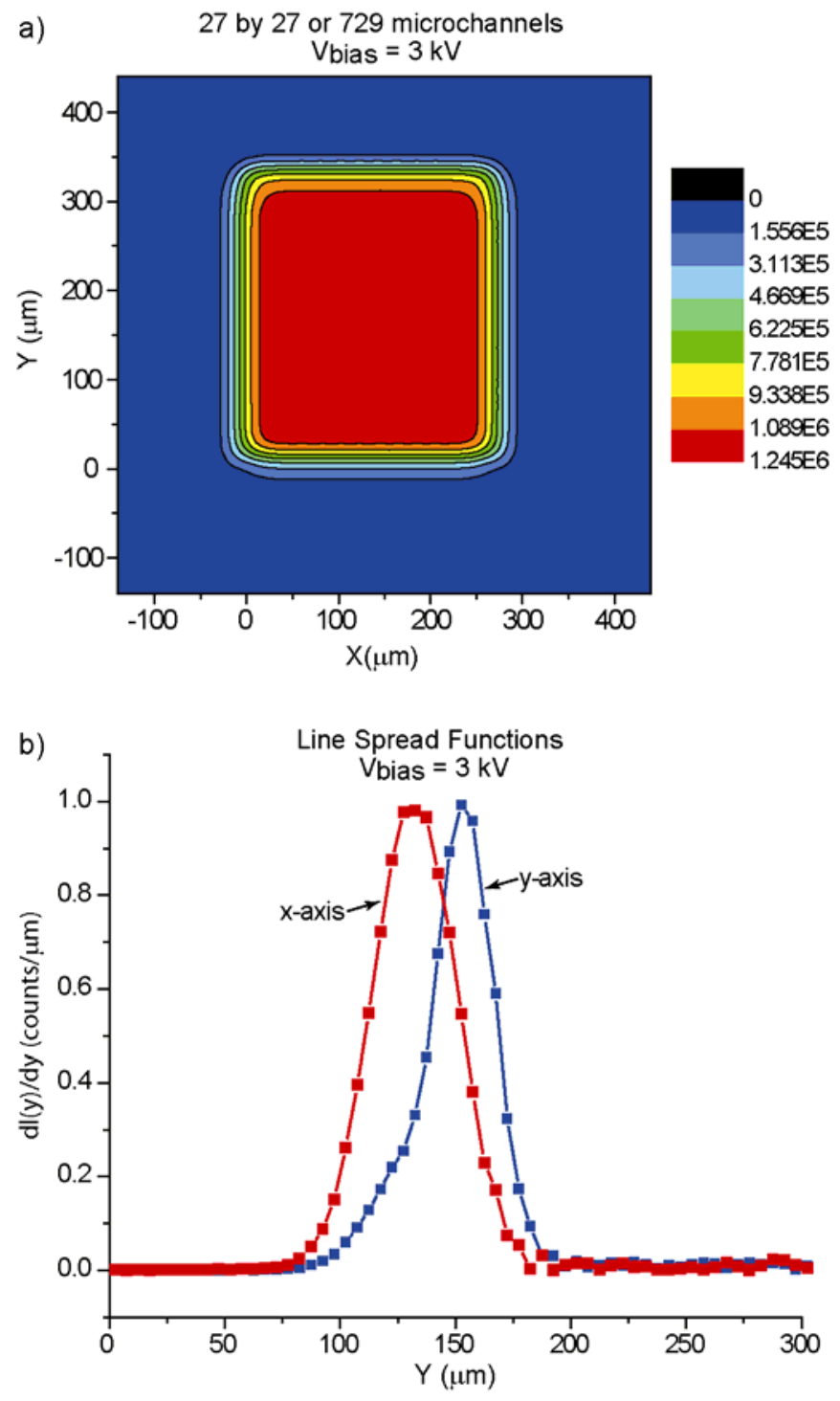

Figure 4. Wu 


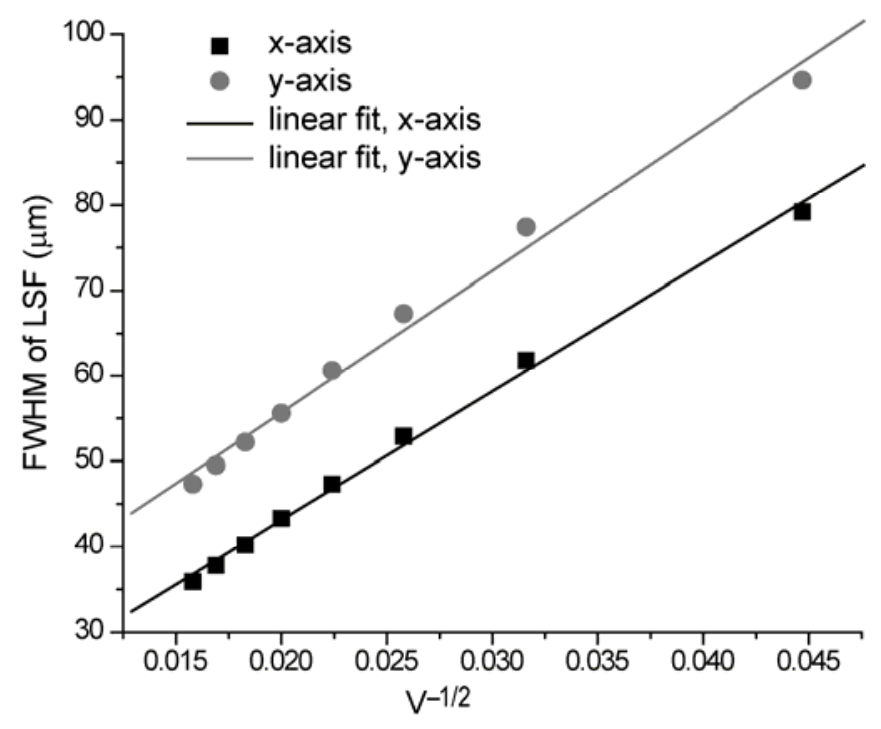

Figure 5. Wu 

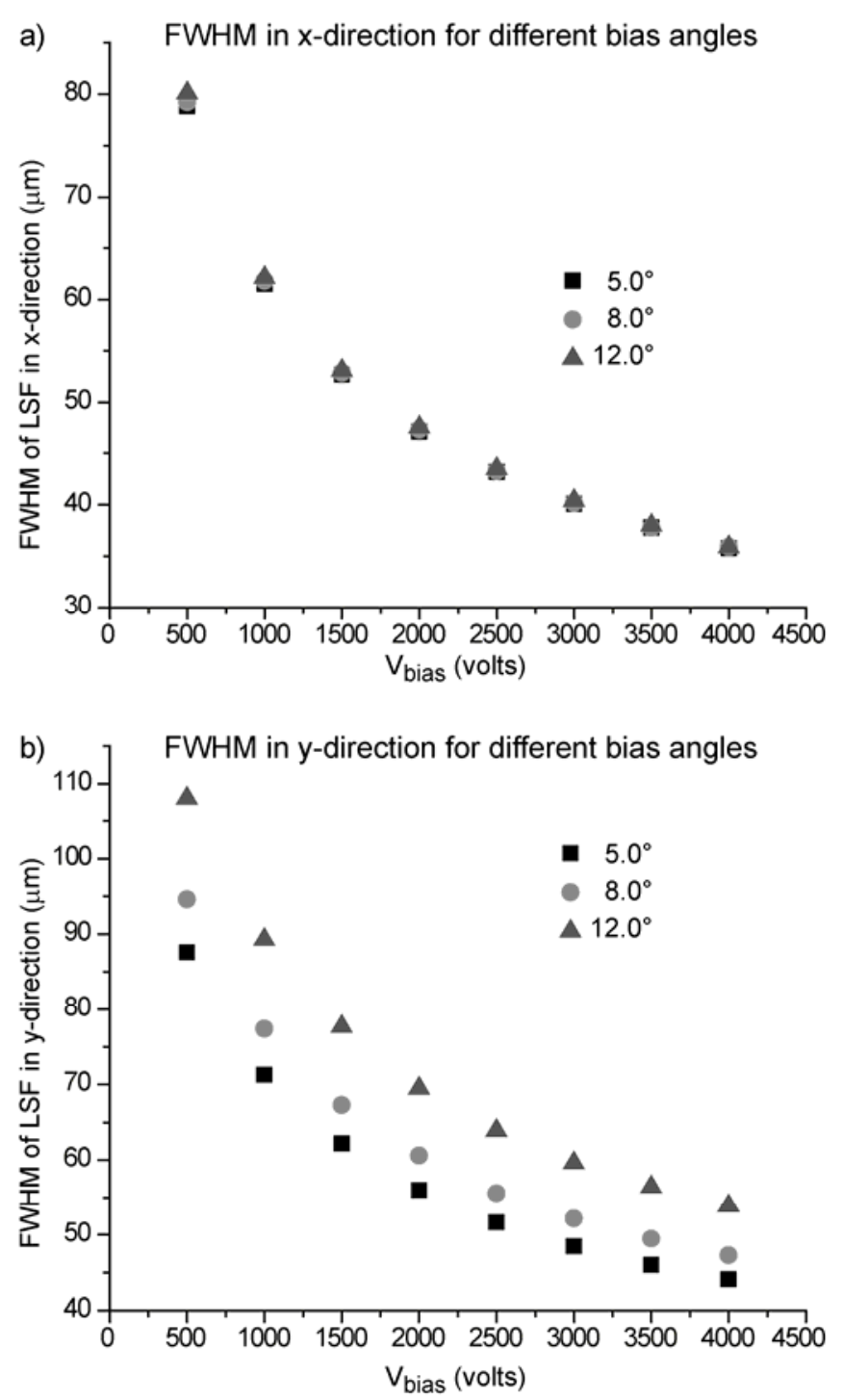

Figure 6. Wu 


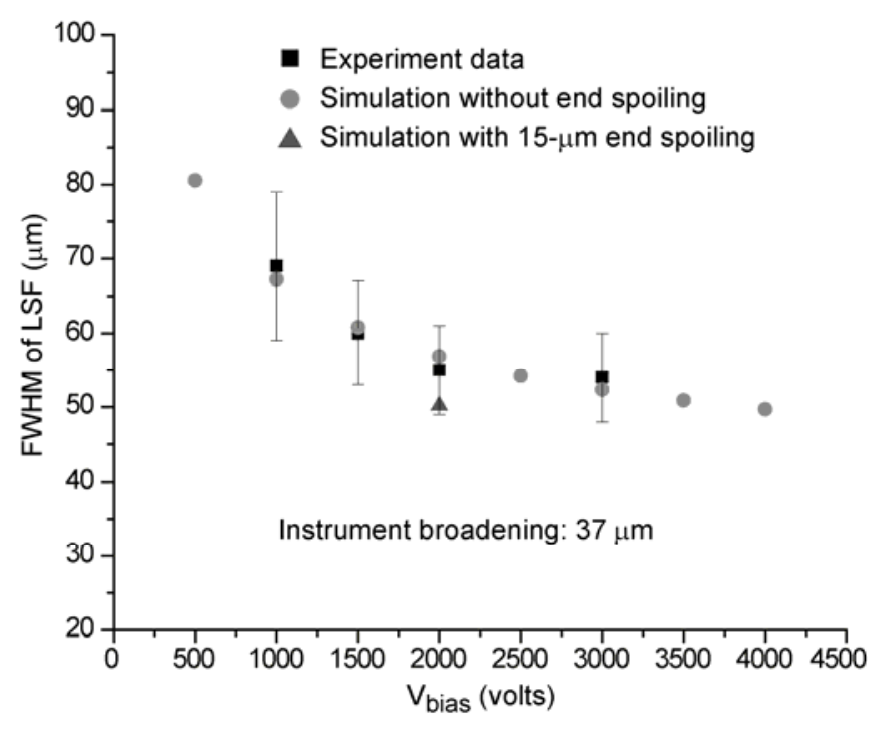

Figure 7. Wu 